\title{
Eficiência dos municípios da Região Norte Fluminense no uso dos recursos públicos: uma análise de 2011 a 2016
}

\author{
Efficiency of Municipalities in the Northern Region of the State of Rio de Janeiro in \\ the Use of Public Resources: an Analysis from 2011 to 2016
}

Resumo: Estudos apontam que o estado não tem sido eficiente na geração de serviços públicos. Essa situação é ainda mais preocupante quando se trata de uma região que recebe elevadas parcelas de orçamento público oriundas de impostos, transferências federais ou royalties de alguma atividade extrativista, como é o caso dos municípios da Região Norte Fluminense, que tem a atividade petrolífera como motor da sua economia. Neste sentido, o objetivo deste artigo consistiu em mensurar e analisar a eficiência dos municípios da Região Norte Fluminense quanto ao uso dos recursos públicos de 2011 a 2016. Realizou-se revisão de literatura e discussão teórica sobre o tema, e, como metodologia, adotou-se a análise envoltória de dados em janelas. Os principiais resultados revelaram que São Fidélis, Macaé e São Francisco de Itabapoana foram eficientes em, pelo menos, um dos anos e apontaram redução no indicador de desenvolvimento dos municípios ineficientes e aumento nos eficientes.

Palavras-chave: Gasto. Público. IFDM. Modelo DEA. Região Norte Fluminense do estado do Rio de Janeiro. Royalties. Petróleo.
Abstract: Studies show that the state has not been efficient in generating public services. It is particularly worrying when it comes to a region that receives high amounts of public budget from taxes, federal transfers or royalties from some extractive activities, as are the municipalities in the Northern of the state of Rio de Janeiro, Brazil, which has the oil activity as the engine of its economy. The objective of this article was to measure and analyze the efficiency of the municipalities of the Northern state of Rio de Janeiro regarding the use of public resources from 2011 to 2016. A literature review and theoretical discussion on the topic were carried out and as methodology, the Data Envelopment Analysis (DEA) was adopted. whether windowed data wrap analysis. The main results revealed that São Fidelis, Macaé and São Francisco de Itabapoana were efficient in at least one of the years and showed a reduction in the development indicator of inefficient municipalities and an increase in efficient ones.

Keywords: Spending. Public. IFDM. DEA model. Northern region of the state of Rio de Janeiro. Royalties. Oil.

\section{Graciela Aparecida Profeta}

Doutorado em Economia Aplicada. Universidade Federal Fluminense. graciela_profeta@yahoo.com.br.

(22) 99601-6333. Rua João Manoel de Abreu, 101, casa 1, Parque Imperial, CEP: 28022-280

Campos dos Goytacazes, RJ

\section{Samuel Alex Coelho Campos}

Doutorado em Economia Aplicada. Universidade Federal Fluminense. samuelcampos@id.uff.br.

(22) 99734-3943. Rua Antuérpio Soares Yong, 203, apto. 101, Parque Flamboyant, CEP 28.015-220

Campos dos Goytacazes, RJ

\section{Priscila da Silva Barroso}

Bacharel em Ciências Econômicas. Universidade Federal Fluminense. pribarrosos@gmail.com.

(22) 997468490. Rua Ramiro Martins Viana, 19, casa 3, Parque Jardim Aeroporto, CEP: 28093020

Campos dos Goytacazes, RJ

\section{Vanuza da Silva Pereira Ney}

Doutorado em Produção Vegetal. Universidade Estadual do Norte Fluminense Darcy Ribeiro. vanuzaney@gmail.com. (22) 99995-4445. Rua Gilberto Siqueira, 60, apto. 702. Centro, CEP: 28010-400

Campos dos Goytacazes, RJ 


\section{Introdução}

Com a atividade petrolífera e a descoberta do pré-sal, muitas empresas foram atraídas para a Região Norte Fluminense (NF) do estado do Rio de Janeiro (ERJ), gerando importantes retornos financeiros e contribuindo para que, nos últimos anos, o PIB per capita do NF tenha se apresentado como o maior do estado.

De acordo com Fundação Centro Estadual de Estatísticas, Pesquisas e Formação de Servidores Públicos do Rio de Janeiro (CEPERJ) (2012), considerando dados de 2010, o PIB do NF representava 11,2\% do PIB estadual. Entretanto, devido aos efeitos negativos de crises econômicas enfrentadas pelo País, como as crises de 2008 e 2012, 2010 e 2016, observou-se uma redução na taxa de crescimento do PIB do País e também do NF e uma forte sinalização de recessão econômica, o que afeta todos os setores da economia.

Associados aos problemas econômicos e políticos, têm-se também os sociais. Segundo o Instituto Brasileiro de Geografia e Estatística (IBGE), com a retração do PIB no Brasil e 0 aumento da população, o PIB per capita teve uma redução ao longo de três anos. Dessa forma, a população perdeu poder de compra, reduzindo a circulação de moeda na economia, gerando um efeito cascata, que culminou em números elevados do desemprego, que apresentou, em 2018, uma taxa média de desocupação de 12,3\%, a maior dos últimos sete anos em 13 capitais do País (IBGE, 2019).

Nota-se, também, que nem sempre o estado é eficiente no uso dos recursos públicos obtidos, o que acaba prejudicando a população, que, por muitas vezes, ou por meio de elevada carga tributária e/ou recursos próprios, arca com despesas para usufruírem de serviços básicos como os relacionados à saúde, educação, infraestrutura e segurança. Essa situação se agrava e, portanto, torna-se ainda mais importante discuti-la quando se trata de estados/ municípios que recebem somas consideráveis de recursos oriundos das atividades econômicas ligadas aos recursos naturais, como é o caso de algumas cidades do ERJ, como São João da Barra, Campos dos Goytacazes e Macaé, que recebem significativas somas de receitas geradas a partir dos royalties da exploração de petróleo.

Ao comparar o Índice FIRJAN de Desenvolvimento Municipal (IFDM) com o PIB per capita para os nove municípios do NF, considerando dados de 2016, observa-se que, embora São João da Barra apresentasse o maior PIB per capita do NF, estava em segundo lugar no ranking em relação ao IFDM e em quinto quando se verificam os valores dos royalties recebidos, apresentando $39 \%$ de suas receitas oriundas dos royalties. Da mesma forma, Quissamã, segunda colocada no ranking do PIB per capita, estava na quarta posição quando se observa o IFDM e na $12^{\mathrm{a}}$ posição em relação aos valores dos royalties recebidos, sendo que o município tinha $28 \%$ de suas receitas compostas desse recurso.

É importante destacar também que Carapebus tinha, em 2016, 32\% de suas receitas oriundas dos royalties do petróleo. No caso de Campos dos Goytacazes e Macaé, ambos apresentavam 15\% de suas receitas oriundas dos royalties e ocupavam, respectivamente, a primeira e segunda posições no ranking em relação aos valores recebidos dos royalties.

Sendo assim, questiona-se: os municípios do NF foram eficientes no uso dos recursos públicos no período de 2011 a 2016? Acredita-se que uma análise sobre a eficiência do uso desses recursos públicos para esses municípios possa contribuir para identificar quais deles têm se destacado quanto ao melhor uso dos recursos e quais precisam tomar medidas que visem garantir a melhoria da eficiência.

A escolha do período de análise se deve ao fato de que pelo Brasil ter passado por um processo de desaceleração na economia brasileira em meados de 2011 e em 2014, ocorreu um agravamento desse processo ocasionando assim uma recessão. Além disso, ressalta-se que o dado mais recente do IFDM data de 2016. Também não se pode deixar de considerar a mudança nas leis dos royalties no que tange à sua distribuição entre estados e municípios. Esta alteração oficializou-se em 2012 com a Lei 12.734/12, a qual redefiniu a distribuição das porcentagens destinadas aos municípios que produzem e aos que não produzem.

\section{Análise de eficiência do uso de recursos púbicos}

São vários os estudos que analisaram a eficiência governamental em gerar serviços púbicos à população utilizando a Análise Envoltória de Dados (DEA), como os de Delgado e Machado (2007); Faria, Januzzi e Silva (2008); Trompieri Neto et al. (2009); Antoun Netto e Lins (2012); Doumpos e Cohen (2014); Lourenço et al. (2017); Andrett et al. (2018); Rodrigues e Sousa (2019) e Miguel e Belda (2020), entre outros. 
Faria, Januzzi e Silva (2008) estudaram a eficiência dos gastos públicos nos municípios fluminenses no período 1999/2000, usando como inputs o gasto per capita com saúde, saneamento e educação. Com relação aos outputs, utilizaram a taxa de alfabetização de 10 a 14 anos; a proporção de domicílios particulares permanentes com esgotamento sanitário adequado; a proporção de domicílios particulares permanentes com saneamento adequado; o inverso da taxa de mortalidade por causas hídricas; e a proporção de crianças de dois a cinco anos matriculadas em creches ou escolas de educação infantil. Os resultados permitiram observar que uma maior eficiência dos gastos proporcionou resultados melhores nas políticas públicas, de forma que os municípios considerados unidades de referência refletiram as boas práticas de gestão ao obterem melhores resultados com menos recursos.

Trompieri Neto et al. (2009) analisaram os determinantes da eficiência dos gastos públicos municipais em saúde e educação no estado do Ceará. Nesse estudo, com relação à função saúde, por exemplo, os insumos selecionados foram o gasto com saúde per capita. E os indicadores de produto selecionados foram: número de unidades de saúde per capita; número de profissionais de saúde com nível superior per capita; número de agentes comunitários de saúde per capita; e taxa de cobertura do Programa Saúde da Família (PSF). A partir dos resultados encontrados, observou-se que "os municípios eficientes em transformar insumo em produto não necessariamente são eficientes na transformação de insumo em resultado, e vice-versa" (TROMPIERI NETO et al., 2009, p. 14).

Antaun Netto e Lins (2012) avaliaram indicadores de desempenho das capitais e do distrito federal na área da saúde, utilizando dados de mortalidade para causas externas; doenças circulatórias; e mortalidade infantil. Os resultados indicaram que as ineficiências obtidas e a determinação dos valores ótimos permitem aos respectivos municípios localizarem as fontes de ineficiência, possibilitando identificar ações para reduzi-las.

Doumpos e Cohen (2014) analisaram a efıciência na administração do setor público de um grupo de municípios gregos, com base em indicadores econômico-financeiros referentes ao período de 2002 a 2009. As estimativas de eficiência foram analisadas em um modelo de regressão. A pesquisa contribuiu para introduzir um novo modelo de avaliação da eficiência dos governos locais na Grécia, por meio da alocação dos dados em inputs e outputs, propiciando uma visão abrangente do orçamento e facilitando a implementação de programas de redução de custos.
Lourenço et al. (2017) analisaram a eficiência técnica dos 250 maiores municípios brasileiros em termos de alunos matriculados no ensino fundamental. Para tanto, usaram como variáveis o Índice de Desenvolvimento da Educação Básica (Ideb) sob a contrapartida de despesas liquidadas; os gastos médios por alunos; e o Índice de Desenvolvimento Humano Municipal (IDHM) dos municípios nas dimensões educação e renda.

Os resultados indicaram que 13 municípios (5,20\% dos 250 maiores em termos de matrículas) foram considerados eficientes. Ao observar o grupo composto pelos 15 municípios com piores desempenhos de eficiência técnica, os autores evidenciaram que, ceteribus paribus, seria necessário um incremento médio na nota média do Ideb na ordem de $90 \%$ para estes serem elevados ao grupo dos municípios considerados eficientes.

Também na área de educação, Rodrigues e Sousa (2019) avaliaram a eficiência dos gastos públicos municipais em educação básica no Brasil. Constataram que elevados PIBs não necessariamente garantem melhores níveis de eficiência. Em relação aos grupos populacionais, observou-se que os municípios com menos de 20.000 habitantes conseguiram alcançar melhores níveis de eficiência.

Em 2020, pode-se citar como exemplo o estudo realizado por Miguel e Belda, que analisaram diversas formas de gestão de hospitais públicos em uma área de gestão homogênea, representativa e altamente complexa, como a administrada pelo Serviço de Saúde de Madrid (SERMAS). Os principais resultados evidenciaram maior eficiência técnica das formas de gestão de hospitais públicos com base na colaboração público-privada em comparação à gestão pública tradicional, que, no entanto, obteve resultados superiores em eficiência de pesquisa do que os obtidos pelas formas de gestão de hospitais baseados em colaboração público-privada.

\section{A intervenção do estado e a eficiência na gestão pública}

A sociedade, as instituições e até mesmo o governo têm grande interesse em saber quais são as atividades desenvolvidas no País, visto que o governo tem como uma de suas funções primordiais prestar serviços que satisfaçam às necessidades coletivas de forma eficiente e eficaz. Além disso, também cabe ao estado o papel de regular a prestação desses serviços, quando são ofertados por terceiros, "geralmente concessionários", 
que são empresas privadas que ganham, em processos licitatórios, o direito à exploração de um bem público.

De acordo com Ferreira (2007), o gestor público deve compreender que o processo de transformação da sociedade é necessário e inevitável, e isso afeta o modo de se gerenciar instituições, departamentos e órgãos públicos. Então, os conceitos de eficiência e eficácia geralmente estão mais presentes na área da administração e da economia. A importância da eficiência e da eficácia na gestão pública tem como base o desenvolvimento social, que afeta diretamente a sociedade como um todo, contribuindo na melhoria dos serviços públicos prestados à sociedade.

De acordo com a Constituição de 1988, mais precisamente 0 artigo 37, os gestores públicos devem agir em nome da administração pública, atuando devidamente respaldados em prévia determinação legal. Isso para garantir que a prestação de serviços seja feita com qualidade, regularidade, transparência e segurança na utilização dos recursos públicos.

Do ponto de vista conceitual e econômico, a eficiência deve ser vista a partir dos estudos de Pareto (1984), Kaldor (1939) e Hicks (1939). Segundo Pareto, a eficiência é atingida quando não se podem gerar melhorias para um dado agente sem que o outro seja prejudicado. Para Kaldor e Hicks, existe a possibilidade de melhoria na eficiência para além do ótimo de Pareto desde que se tenham outros arranjos produtivos, institucionais, pessoais etc., que, mesmo que prejudiquem terceiros, estes possam ser recompensados.

Assim, na gestão pública, os princípios da eficiência e eficácia têm como direção o desenvolvimento público relacionado ao impacto social, que procura identificar os efeitos produzidos sobre uma população que vive de programas sociais desenvolvidos pelos governos federal, estadual e municipal.

Segundo Keynes (1964), a intervenção estatal apresenta-se, principalmente, a partir da política fiscal, que se ampara tanto na administração dos gastos públicos quanto na política de tributação. Além disso, na visão keynesiana, a política fiscal recai diretamente sobre a demanda agregada, que, por consequência, leva ao investimento e ao consumo público e privado.

Pereira (2006, p. 52) afirma que a política fiscal "refere-se, por um lado, às receitas públicas, [...]; e, por outro lado, os dispêndios do governo, os quais estão explicitados no orçamento público". Dessa forma, a política fiscal abrange dois componentes distintos, o relativo à política tributária, concernente à receita pública, e a política orçamentária, pertinente à despesa pública.

Por fim, segundo o próprio Keynes (1964), as políticas monetárias e/ou fiscais devem ser dosadas não somente para que seus efeitos não sejam adversos em relação aos objetivos da intervenção estatal, mas, principalmente, porque a política econômica é uma norma, uma convenção, que orienta a tomada de decisão dos empresários. Então, a possibilidade de intervenção governamental pode gerar uma possibilidade do questionamento da efetividade desta.

\section{Metodologia}

A análise da eficiência pode ser operacionalizada por meio de métodos não paramétricos ou métodos paramétricos. Na análise paramétrica, tem-se a Análise de Fronteira Estocástica (SFA), enquanto, entre os métodos não paramétricos, cita-se a Análise Envoltória de Dados (DEA).

A DEA possui como benefício em relação à SFA a não utilização de uma forma funcional; contudo, considera variações aleatórias da produção como ineficiência, enquanto a SFA acomoda essas variações aleatórias em um termo de erro, não sendo consideradas ineficiências; mas necessita de uma forma funcional explícita (SIPILÄINEN e HUHTALA, 2013). Entretanto, essas técnicas ranqueiam, de forma similar, as unidades tomadoras de decisão analisadas (DMU, sigla em inglês) (FERRIER e LOVELL, 1990; REINHARD, LOVELL e THIJSSEN, 2000).

Entre os diversos modelos de cálculo da eficiência, podem-se citar os propostos por Charnes, Cooper e Rhodes (1978), Banker, Charnes e Cooper (1984). A partir desses modelos, foram propostas outras variações, como Tone (2001; 2004).

Os modelos propostos por Charnes, Cooper e Rhodes (1978) e Banker, Charnes e Cooper (1984) permitem que, em função da fronteira de produção poliangular estimada, mesmo uma DMU com folga no uso dos insumos seja considerada eficiente. Por outro lado, o Modelo de Eficiência com Medidas Baseada nas Folgas proposto por Tone (2001; 2004) incorpora à análise possíveis folgas no uso dos insumos ou da produção, sendo um método de maior capacidade de discriminação entre DMUs eficientes e ineficientes. Assim, conforme Tone 
(2001), a eficiência técnica das DMUs pode ser calculada por meio da Equação (1):

$$
\begin{aligned}
& \min \tau_{1}=t-\frac{1}{m} \sum_{i=1}^{m} s_{i}^{-} / x_{i o} \\
& \text { s.a. } \\
& 1=t+\frac{1}{s} \sum_{r=1}^{s} s_{r}^{+} / y_{r o} ; t x_{o}=X \Delta+S^{-} ; \quad y_{o}=Y \Delta-S^{+} ; \Delta \geq 0, \quad S^{-} \geq 0, \quad S^{+} \geq 0 e t>0
\end{aligned}
$$

em que o termo " $X$ " denota a matriz de insumos; " $Y$ " é a matriz de produtos; e " $s$ " e "s" indicam o excesso no uso dos insumos e o déficit da produção, respectivamente; " $\lambda$ " é o vetor de intensidade, " $t$ ", um escalar positivo; e " $\tau_{1}$ " é o vetor dos escores de eficiência técnica calculado para cada DMU, que assume valores entre 0 e 1. A eficiência sobre a pressuposição de retornos variáveis à escala pode ser calculada adicionando a restrição $e \lambda=\sum_{j=1}^{n}=1$ à Equação (1) (TONE, 2001).

Neste artigo, utilizaram-se como insumos (inputs): (i) investimento per capita na oferta de serviços e produtos para cada município; (ii) despesa total municipal per capita; (iii) royalties per capita - denota o valor recebido dos royalties para cada município. Os dados de insumos foram obtidos com o Tribunal de Contas do Estado do Rio de Janeiro (TCE/RJ) (2016).

Como produtos (outputs), utilizou-se o índice FIRJAN de desenvolvimento humano municipal (Federação das Indústrias do Estado do Rio de Janeiro, 2019), que leva em consideração dados de saúde, educação, longevidade e renda. Outros estudos utilizaram variáveis semelhantes, como o de Doumpos e Cohen (2014).

Em função do período de análise considerar uma série temporal de 2011 a 2016, foi implementado o método de Análise de Janela (DEA Window Analysis). Esse método permite que a tendência e estabilidade da eficiência de cada DMU seja analisada ao longo do tempo (CHARNES e COOPER, 1985). Então, há uma combinação da análise cross-section com série temporal, como proposto por
Charnes et al. (1984).

A Análise de Janela é um método análogo a uma média móvel, em que cada DMU, em cada ano é tratada como uma DMU diferente, comparando a performance de uma DMU no período t às demais DMUs dos demais períodos j, para todo $j \neq t$, (COOPER, SEIFORD e ZHU, 2011).

0 método forma janelas incluindo apenas alguns anos a cada momento, e calculam-se os respectivos escores de eficiência. Assim, considerando os anos analisados nesse estudo (2011 a 2016) e baseando-se em Halkos e Tzeremes (2009), foram definidas quatro janelas com o respectivo período $J_{1}\left(2011,2012\right.$ e 2013); $J_{2}$ (2012, 2013 e 2014); J $J_{3}\left(2013,2014\right.$ e 2015); e $J_{4}(2014,2015$ e 2016). Logo, definido o tamanho da janela, em anos, e 0 ano inicial, forma-se a janela 1 (um). Para a janela dois, inclui-se um ano adjacente e retira-se 0 ano anterior e assim sucessivamente, de modo que a Equação (1) foi calculada separadamente para cada uma das janelas.

\section{Resultados e discussões}

Inicialmente, aplicou-se o teste de Kolmogorov-Smirnov para testar a hipótese nula de ausência de (in)eficiência de escala, o que implica calcular a eficiência usando o modelo com a pressuposição de retornos constantes, contra a hipótese alternativa de presença de (in) eficiente de escala. 0 teste foi implementado para cada janela, sendo estes os valores calculados: $D=0,40741$; $D=0,40741 ; D=0,33333 ;$ e $D=0,25926$ para as janelas 1, 2, 3 e 4, respectivamente.

A partir desses resultados, pôde-se rejeitar a hipótese nula no nível de significância de 1\%. Com isso, calculou-se eficiência das DMUs sobre a pressuposição de retornos variáveis. Então, na Figura 1, apresentam-se os escores médios de eficiência para o período de 2011 a 2016 calculados por meio da análise em janela.




Figura 1- Escores médios de eficiência para os municípios do NF, 2011 a 2016.

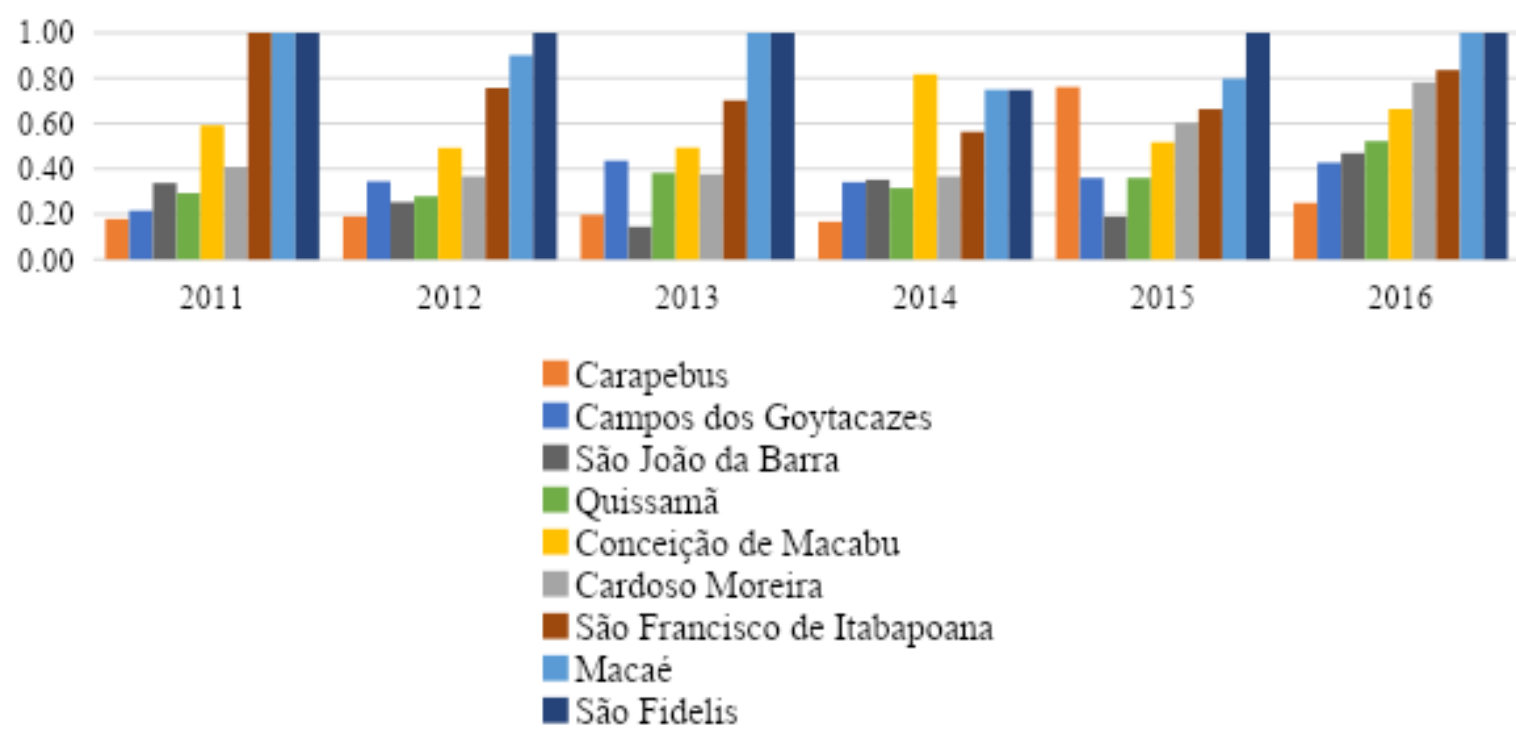

Fonte: Elaboração própria a partir dos resultados da pesquisa.

A eficiência média indicou que os municípios de São Fidélis, Macaé e São Francisco de Itabapoana foram eficientes em, pelo menos, um dos anos analisados. Os demais municípios foram ineficientes quanto a investimentos, despesas e uso dos royalties na produção de desenvolvimento municipal (IFDM) em todos os anos analisados.

Salienta-se que São João da Barra e Campos dos Goytacazes, primeiro e quarto colocados no ranking quanto ao PIB per capita e quinto e primeiro em relação ao volume de receitas oriundas dos royalties não se mostraram eficientes, pelo contrário, apresentaram valores de eficiência bem menores do que os de Conceição de Macabu, por exemplo, que ocupava, em 2016, a 62 a posição no ranking de receitas recebidas com royalties. Logo, os resultados corroboram aqueles obtidos por Rodrigues e Sousa (2019) no que tange à relação PIB-eficiência técnica do gasto público.

Destaca-se que, em 2014, nenhum dos municípios foi eficiente. Tal resultado pode ter ocorrido em função dos efeitos da mudança na Leis dos royalties em 2012, que redistribuiu os recursos oriundos dos royalties, podendo ter gerado desequilíbrios nos orçamentos públicos dos municípios para os anos subsequentes, uma vez que os efeitos de mudanças em Leis não são observados de imediato, mas sim em períodos futuros à sua implementação. Outro fator que pode ter levado aos resultados apresentados em 2014 diz respeito ao fato de os municípios terem antecipados recursos dos royalties em 2013 e em 2014. Ribeiro, especialista em finanças públicas, em entrevista a Duarte (2014), para o Jornal Tarde, alegou que a antecipação dos royalties poderia causar problemas aos futuros governos, uma vez que haveria comprometimento das receitas recebidas para orçamentos futuros.

No que se refere aos resultados dos escores de eficiência para cada ano por município e por janela, estes estão apresentado na Tabela 1.
UNIVERSIDADE CANDIDO MENDES

\section{A QUALIDADE DE ENSINO QUE VOCÊ JÁ CONHEGE.}


Tabela 1- Escores de eficiência por município para as janelas de 1 a 4 e valor médio, 2011 a 2016

\begin{tabular}{|c|c|c|c|c|c|c|}
\hline Município & Ano & J1 & $\mathbf{J 2}$ & J3 & J4 & Média \\
\hline \multirow{6}{*}{ Campos dos Goytacazes } & 2011 & 0,21 & & & & 0,21 \\
\hline & 2012 & 0,34 & 0,34 & & & 0,34 \\
\hline & 2013 & 0,44 & 0,44 & 0,44 & & 0,44 \\
\hline & 2014 & & 0,33 & 0,33 & 0,36 & 0,34 \\
\hline & 2015 & & & 0,29 & 0,43 & 0,36 \\
\hline & 2016 & & & & 0,43 & 0,43 \\
\hline \multirow{6}{*}{ Carapebus } & 2011 & 0,18 & & & & 0,18 \\
\hline & 2012 & 0,19 & 0,19 & & & 0,19 \\
\hline & 2013 & 0,18 & 0,20 & 0,20 & & 0,20 \\
\hline & 2014 & & 0,15 & 0,15 & 0,20 & 0,17 \\
\hline & 2015 & & & 1,00 & 0,52 & 0,76 \\
\hline & 2016 & & & & 0,25 & 0,25 \\
\hline \multirow{6}{*}{ Cardoso Moreira } & 2011 & 0,41 & & & & 0,41 \\
\hline & 2012 & 0,34 & 0,39 & & & 0,37 \\
\hline & 2013 & 0,35 & 0,39 & 0,39 & & 0,38 \\
\hline & 2014 & & 0,32 & 0,32 & 0,46 & 0,37 \\
\hline & 2015 & & & 0,58 & 0,62 & 0,60 \\
\hline & 2016 & & & & 0,78 & 0,78 \\
\hline \multirow{6}{*}{ Conceição de Macabu } & 2011 & 0,59 & & & & 0,59 \\
\hline & 2012 & 0,46 & 0,52 & & & 0,49 \\
\hline & 2013 & 0,45 & 0,51 & 0,51 & & 0,49 \\
\hline & 2014 & & 0,72 & 0,72 & 1,00 & 0,82 \\
\hline & 2015 & & & 0,51 & 0,52 & 0,52 \\
\hline & 2016 & & & & 0,66 & 0,66 \\
\hline \multirow{6}{*}{ Macaé } & 2011 & 1,00 & & & & 1,00 \\
\hline & 2012 & 0,80 & 1,00 & & & 0,90 \\
\hline & 2013 & 1,00 & 1,00 & 1,00 & & 1,00 \\
\hline & 2014 & & 1,00 & 0,76 & 0,48 & 0,75 \\
\hline & 2015 & & & 1,00 & 0,59 & 0,80 \\
\hline & 2016 & & & & 1,00 & 1,00 \\
\hline \multirow{6}{*}{ Quissamã } & 2011 & 0,29 & & & & 0,29 \\
\hline & 2012 & 0,28 & 0,28 & & & 0,28 \\
\hline & 2013 & 0,38 & 0,38 & 0,38 & & 0,38 \\
\hline & 2014 & & 0,33 & 0,33 & 0,27 & 0,31 \\
\hline & 2015 & & & 0,32 & 0,40 & 0,36 \\
\hline & 2016 & & & & 0,52 & 0,52 \\
\hline \multirow{6}{*}{ São Fidélis } & 2011 & 1,00 & & & & 1,00 \\
\hline & 2012 & 1,00 & 1,00 & & & 1,00 \\
\hline & 2013 & 1,00 & 1,00 & 1,00 & & 1,00 \\
\hline & 2014 & & 0,62 & 0,62 & 1,00 & 0,75 \\
\hline & 2015 & & & 1,00 & 1,00 & 1,00 \\
\hline & 2016 & & & & 1,00 & 1,00 \\
\hline \multirow{6}{*}{ São Francisco de Itabapoana } & 2011 & 1,00 & & & & 1,00 \\
\hline & 2012 & 0,72 & 0,80 & & & 0,76 \\
\hline & 2013 & 0,68 & 0,71 & 0,71 & & 0,70 \\
\hline & 2014 & & 0,56 & 0,56 & 0,57 & 0,56 \\
\hline & 2015 & & & 0,67 & 0,65 & 0,66 \\
\hline & 2016 & & & & 0,83 & 0,83 \\
\hline \multirow{6}{*}{ São João da Barra } & 2011 & 0,34 & & & & 0,34 \\
\hline & 2012 & 0,25 & 0,25 & & & 0,25 \\
\hline & 2013 & 0,14 & 0,14 & 0,14 & & 0,14 \\
\hline & 2014 & & 0,36 & 0,36 & 0,34 & 0,35 \\
\hline & 2015 & & & 0,19 & 0,19 & 0,19 \\
\hline & 2016 & & & & 0,47 & 0,47 \\
\hline
\end{tabular}

Fonte: Elaboração própria a partir dos resultados da pesquisa. 
Na Tabela 1, as linhas permitem que seja analisada a estabilidade da eficiência técnica dos municípios ao longo das diferentes janelas, enquanto as colunas indicam 0 comportamento observado de um mesmo município em uma mesma janela. Então, tomando Campos dos Goytacazes como exemplo, observa-se a estabilidade dos escores de eficiência ao longo das janelas, visto que, para 2013, em que foram calculadas a eficiência desse município nas janelas $J_{1}, J_{2}$ e $J_{3^{\prime}}$ os escores de eficiência foram iguais a 0,44 nas três janelas supracitadas.

Entre os municípios do NF, observa-se tendência de aumento da eficiência no uso dos insumos para o desenvolvimento econômico, visto que os escores médios aumentaram entre 2011 e 2016, exceto para São Francisco de Itabapoana. Em 2011, o município apresentou escore de 1,00 (100\% eficiente), enquanto, em 2016, o escore reduziu para 0,83, (-13 p.p). Por outro lado, Campos dos Goytacazes, Carapebus, Cardoso Moreira, Conceição de Macabu, Quissamã e São João da Barra aumentaram sua eficiência em 21, 7, 37, 7, 23, e 13 p.p., respectivamente; enquanto Macaé e São Fidélis foram eficientes tanto em 2011 quanto em 2016, mas o primeiro foi ineficiente em 2012, 2014 e 2015 e o segundo, em 2014.

Ademais, essa melhoria na alocação dos recursos disponíveis nos municípios (ou sua piora para o município de São Francisco de Itabapoana) não ocorreu de forma linear, ou seja, há momentos com ganhos no desempenho, seguidos de redução no período subsequente e vice-versa. A Tabela 2 apresenta uma comparação entre o uso de insumos utilizados para a produção do desenvolvimento municipal (IFDM-output) entre 2011 e 2016.

\section{Tabela 3- Comparação entre os municípios eficientes e ineficientes quanto ao IFDM, despesas, royalties e investimentos, 2011 e 2016}

\begin{tabular}{lccc}
\hline Especificação & Eficientes & Ineficientes & Diferença (em \%) \\
\hline & 2011 & & \\
\hline IFDM & 0,722 & 0,698 & $-3,4 \%$ \\
Despesas per capita, em reais & $3.331,07$ & $5.507,63$ & $39,5 \%$ \\
Royalties per capita, em reais & 918,10 & $3.012,73$ & $69,5 \%$ \\
Investimentos per capita, em reais & 275,18 & 514,19 & $46,5 \%$ \\
\hline & 2016 & & \\
\hline IFDM & 0,757 & 0,674 & $-12,4 \%$ \\
Despesas per capita, em reais & $4.905,34$ & $5.523,45$ & $11,2 \%$ \\
Royalties per capita, em reais & 738,80 & $1.150,84$ & $35,8 \%$ \\
Investimentos per capita, em reais & 76,20 & 193,42 & $60,6 \%$ \\
\hline
\end{tabular}

Fonte: Elaboração própria a partir dos resultados da pesquisa.

De acordo com os dados da Tabela 2, os municípios ineficientes apresentaram, em ambos os anos selecionados, IFDM inferior àqueles dos municípios eficientes em $3,4 \%$ e $12,4 \%$, respectivamente, mesmo tendo utilizado, proporcionalmente, uma maior quantidade de insumos para obterem esse IFDM inferior. Tomando as despesas de capital, os municípios ineficientes utilizaram, em 2011 e $2016,39,5 \%$ e $11,2 \%$ a mais do que os municípios eficientes.

Mesmo que esses dados denotam que no período os municípios que se mostraram ineficientes reduziram o desperdício com despesas de capital, ainda assim não os colocou em posição de eficiência. Isso pode ter sido 
em decorrência de terem aumentado seus investimentos de forma inadequada, dispendendo, proporcionalmente, $46,5 \%$ e $60,6 \%$ a mais do que o valor médio dos municípios considerados eficientes.

Os resultados indicam uma redução no indicador de desenvolvimento municipal nos municípios ineficientes, e aumento nos eficientes e uma redução no valor dos royalties e investimentos; mas também um aumento nas despesas de capital, tanto para os eficientes como ineficientes. Assim, os resultados da Tabela 2 corroboram a tendência de ganhos de eficiência representados pela Figura 1, no sentido de que os municípios ineficientes reduziram o desperdício de recursos para promover 0 desenvolvimento municipal: o uso médio dos insumos reduziu (excetuando-se os investimentos) proporcionalmente em relação aos municípios eficientes.

Destaca-se que o método DEA em janela ou mesmo o tradicional considera a proporção produto obtido com 0 uso de insumos. Assim, por exemplo, considerando o município de Campos dos Goytacazes, em 2016, que apresentou um IFDM de 0,72 utilizando $\mathrm{R} \$$ 6.021,4; R\$ 802,33; e R\$ 511,66 com despesas per capita, royalties per capita e investimento per capita, respectivamente, se a ineficiência no uso dos insumos fosse eliminada, esse município poderia obter o mesmo IFDM de 0,72, redu- zindo os insumos supracitados, respectivamente, em aproximadamente, $R \$ 2.961,00, R \$ 316,08$ e $R \$$ 427,50.

\section{Conclusões}

De modo geral, verificou-se que a eficiência média obtida por meio da técnica DEA em janelas indicou que os municípios de São Fidélis, Macaé e São Francisco de Itabapoana foram eficientes em, pelo menos, um dos anos, considerando 2011 a 2016. Quanto aos outros seis municípios da Região Norte Fluminense, observou-se foram ineficientes quanto a investimentos, despesas e uso dos royalties na geração e no aumento do desenvolvimento municipal, mensurado por meio do IFDM em todos os anos analisados.

Além disso, a técnica DEA em janelas mostrou que a promoção do desenvolvimento municipal não ocorreu de forma linear, uma vez que se observaram anos em que houve ganhos no desempenho, seguidos de outros com redução, e vice-versa. Por fim, notou-se que nem sempre municípios com economias mais dinâmicas e maior arrecadação de receitas, como Campos dos Goytacazes e Macaé, foram capazes de ter uma gestão eficiente dos recursos públicos.

\section{A QUALIDADE DE ENSINO QUE VOCE JÁ CONHEGE.}
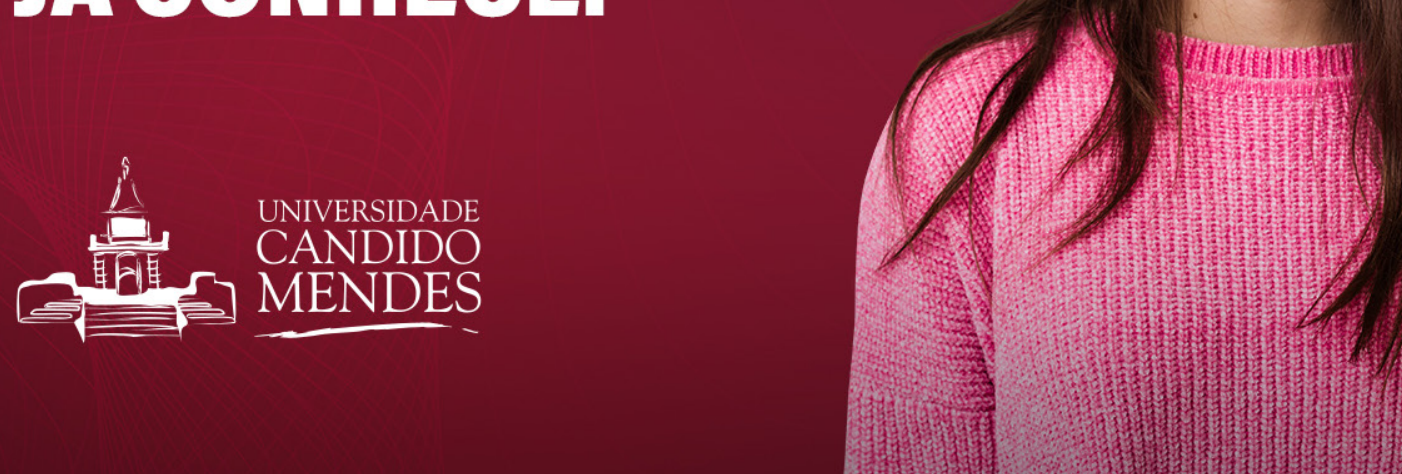


\section{Referências}

ANDRETT, M. C. S. et al. Eficiência dos gastos públicos em saúde no Brasil: estudo sobre o desempenho de estados brasileiros. Revista de Gestão em Sistemas de Saúde, São Paulo, v. 7, p. 114-128, 2018.

ANTOUN NETTO, S. O.,LINS, M. P. E. Análise envoltória de dados (DEA) aplicada na determinação de indicadores de desempenho das capitais e do distrito federal brasileiros na área de saúde. In: CONGRESO LATINO-IBEROAMERICANO DE INVESTIGACIÓN OPERATIVA, 16; SIMPÓSIO BRASILEIRO DE PESQUISA OPERACIONAL, 44, 2012, Rio de Janeiro. Anais[...]. Rio de Janeiro: ILTC, 2012. v. 1. p. 858-867. Disponível em: http://www.din.uem.br/sbpo/sbpo2012/pdf/ arq0238.pdf. Acesso em: 15 fev. 2019.

BANKER, R. D.; CHARNES, A.; COOPER, W. W. Some models for estimating technical ad scale efficiency in data envelopment analysis. Management Science, Catonsville, v. 30, n. 9, p. 1078-1092, 1984.

CEPERJ. Fundação Ceperj divulga PIB dos municípios fluminenses. Rio de Janeiro, 2012. Disponível em: http://www.ceperj.rj.gov.br/noticias/ Dezembro_12/16/PIB_municipal.html. Acesso em: 3 out. 2017.

CHARNES, A.; COOPER, W. W. Preface to topics in data envelopment analysis. Annals of Operations Research, New York, v. 2, n. 1, p. $59-94,1985$.

CHARNES, A. COOPER, W. W; CLARK, C. T; GOLANY, B. A developmental study of data envelopment analysis in measuring the efficiency of maintenance units in the U.S. air forces. Annals of Operations Research, New York, v. 2, p. 95 - 112, 1984.

CHARNES, A.; COOPER, W. W., RHODES, E. D. Measurement the efficiency of decision making units. European Journal of Operational Research, Amsterdam, v. 2, n. 6, p. $429-444,1978$.

DELGADO, V. M. S.; MACHADO, A. F. Eficiência das escolas públicas estaduais de Minas Gerais. Pesquisa e Planejamento Econômico, Rio de Janeiro, v. 37. n. 3, dez. 2007.

DOUMPOS, M.; COHEN, S. Applying data envelopment analysis on accounting data to assess and optimize the efficiency of Greek local governments. Omega, Oxford, v. 46, p. 74-85, 2014.

DUARTE, F. Antecipação dos royalties injeta R\$ 769 mi no Funprev. 2014. Disponível em: https://atarde.uol.com.br/economia/noticias/antecipacao-dos-royalties-injeta-r-769-mi-no-funprev-1609701. Acesso em: 25 maio 2020.

FARIA, F. P.; JANUZZI, P. de M.; SILVA, S. J. da. Eficiência dos gastos municipais em saúde e educação: uma investigação através da análise envoltória no estado do Rio de Janeiro. RAP. Revista Brasileira de Administração Pública, Rio de Janeiro, v. 42, n. 1, p. 155/0034, 2008.

FEDERAÇÃO DAS INDÚSTRIAS DO ESTADO DO RIO DE JANEIRO- FIRJAN. Índice FIRJAN de Desenvolvimento Municipal (IFDM). Disponível em: https:// www.firjan.com.br/ifdm/consulta-ao-indice/. Acesso em: 13 mar. 2019.

FERREIRA, M. K. B. As novas configurações da Gestão Pública: comunicação, conhecimento e pessoas. 2007. Disponível em: https://www3.faac.unesp. br/anais-comunicacao/textos/34.pdf. Acesso em: 20 mar. 2020.

FERRIER, G. D.; LOVELL, C. A. K. Measuring cost efficiency in banking econometric and linear programming evidence. Journal of Econometrics, Amsterdam, n. 46, n. $1-2$, p. 229-245, 1990.

HALKOS, G. E.; TZEREMES, N. G. Exploring the existence of Kuznets curve in countries' environmental efficiency using DEA window analysis. Ecological Economics, Amsterdam, v. 68, n. 7, p. 2168-2176, May 2009.

HICKS, J. The foundations of welfare economics. Economic Journal, Chichester, v. 49, n. 196, p. 696-712, 1939.

KALDOR, N. Welfare Propositions in Economics and Interpersonal Comparisons of Utility. Economic Journal, Chichester, v. 49, n. 195, p. 549-552, 1939.

KEYNES, J. M. The general theory of employment, interest and money. New York: HBJ Book, 1964.

LOURENCO, R. L.; ANGOTTI, M.; NASCIMENTO, J. H. B.; SAUERBRONN, F. F. Eficiência do gasto público com ensino fundamental: uma análise dos 250 maiores municípios brasileiros. Contabilidade Vista \& Revista, Belo Horizonte, v. 28, p. 89-116, 2017.

MIGUEL, J. L. F.; BELDA, C. F. Influence of the management models based on public-private collaboration in the technical and research efficiency of hospitals of the public health system. Revista de Contabilidad - Spanish Accounting Review, Murcia, v. 23, n. 1, p. 249-262, 2020.

PARETO, V. Manual de economia política. Tradução João Guilherme Vargas Neto. São Paulo: Abril Cultural, 1984. (Coleção Os Economistas)

PEREIRA, J. M. Finanças Públicas: A política orçamentária no Brasil. São Paulo: Editora Atlas, 2006.

REINHARD. S.; LOVELL. C. A. K. THIJSSEN, GEERT. J. Environmental efficiency with multiple environmentally detrimental variables; estimated with SFA and DEA. European Journal of Operational Research, Amsterdam, v. 121, n. 2, p.287-303, 2000.

RODRIGUES, A. M. G.; SOUSA, E. P. Eficiência dos gastos públicos em educação básica nos municípios brasileiros. Economic Analysis of Law Review, Taguatinga, v. 10, p. 193-219, 2019 
SIPILÄINEN, T.; HUHTALA, A. Opportunity costs of providing crop diversity in organic and conventional farming: would targeted environmental policies make economic sense? European Review of Agricultural Economics, Oxford, v. 40, n. 3, p. 441-462, July. 2013.

TRIBUNAL DE CONTAS DO ESTADO DO RIO DE JANEIRO (TCE/RJ). Estudos socioeconômicos: municípios do estado do Rio de Janeiro. 2016. Disponível em: https://www.tce.rj.gov.br/estudos-socioeconomicos. Acesso em: 10 mar. 2019

TONE, K. A slacks-based measure of efficiency in data envelopment analysis. European Journal of Operational Research, Amsterdam, v. 130, n. 3, p. 498-509, 2001

TONE, K. Dealing with undesirable outputs in DEA: A slacks-based measure (SBM) approach. GRIPS Research Report Series, Toronto, v. 2004, p. 44-45, Jun 2004

TROMPIERI NETO, N. et al. Determinantes da eficiência dos gastos públicos municipais em educação e saúde: o caso do Ceará. In: CARVALHO, E.B.S. Economia do Ceará em Debate. Fortaleza: IPECE, 2009. p. 57-72. Disponível em http://www.repositorio.ufc.br/bitstream/riufc/2542/1/2008_\%20capliv_ ntrompierineto.pdf. Acesso em: 17 mar. 2020.

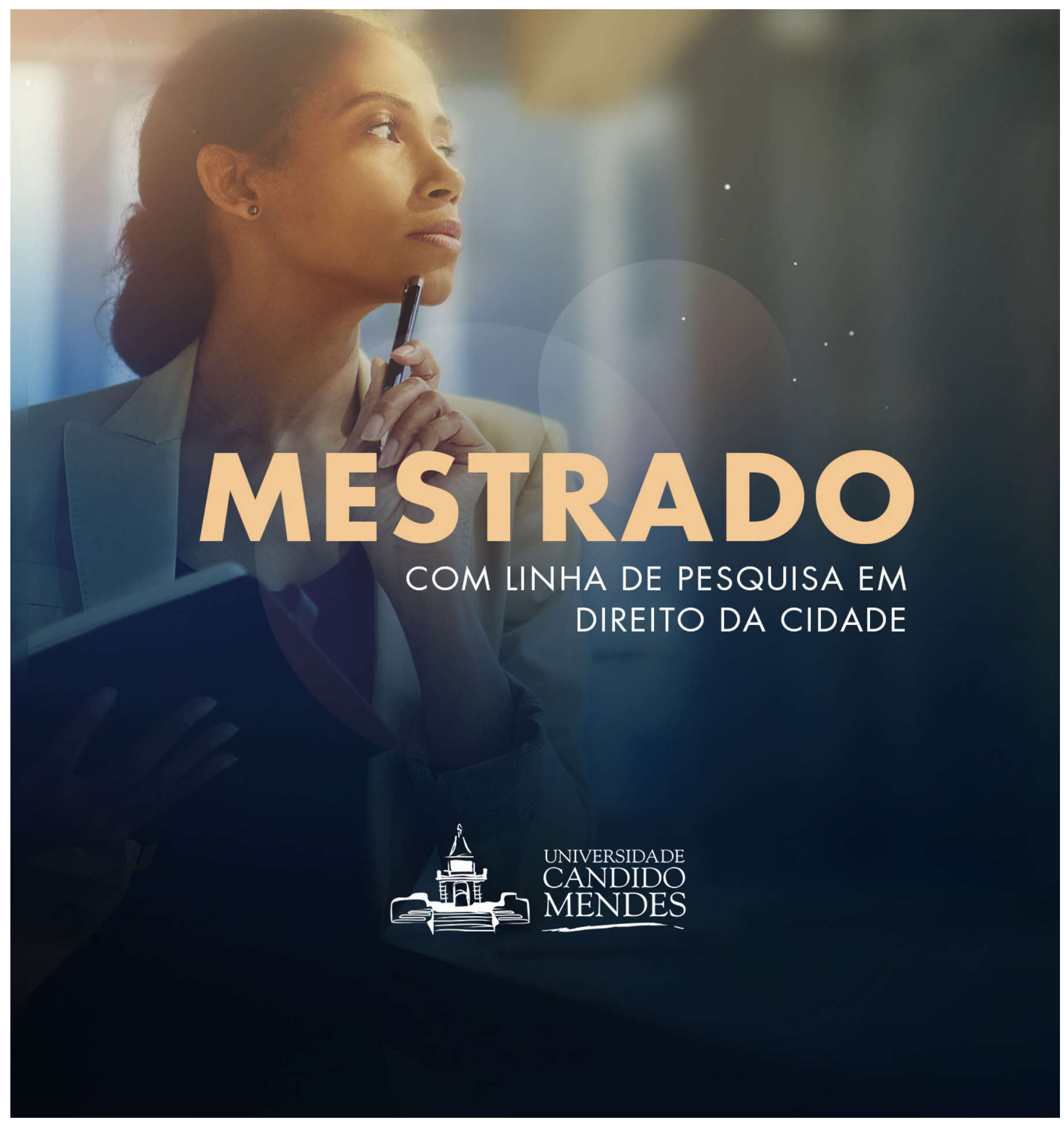

